\title{
A network pharmacology-based study on Alzheimer disease prevention and treatment of Qiong Yu Gao
}

Jie-shu You', Chen-yue Li ${ }^{1}$, Wei Chen ${ }^{1}$, Xia-lin Wu${ }^{2}$, Li-jie Huang ${ }^{1}$, Ren-kai Li ${ }^{3}$, Fei Gao ${ }^{4}$, Ming-yue Zhang ${ }^{5}$, Huan-lan Liu ${ }^{1}$ and Wei-ling Qu ${ }^{1 *}$

* Correspondence: quweiling@ gzucm.edu.cn

${ }^{1}$ School of Basic Medical Sciences, Guangzhou University of Chinese Medicine, Guangzhou, Guangdong Province, China

Full list of author information is available at the end of the article

\begin{abstract}
Background and objective: As the pathological mechanisms of AD are complex, increasing evidence have demonstrated Chinese Medicine with multi-ingredients and multi-targets may be more suitable for the treatment of diseases with complex pathogenesis. Therefore, the study was to preliminarily decipher the bioactive compounds and potential mechanisms of Qiong Yu Gao (QYG) for AD prevention and treatment by an integrated network pharmacology approach.
\end{abstract}

Methods: Putative ingredients of QYG and significant genes of AD were retrieved from public database after screening. Then QYG ingredients target proteins/genes were obtained by target fishing. Compound-target-disease network was constructed using Cytoscape to decipher the mechanism of QYG for AD. KEGG pathway and GO enrichment analysis were performed to investigate the molecular mechanisms and pathways related to QYG for AD treatments.

Results: Finally, 70 compounds and 511 relative drug targets were collected. In which, 17 representative direct targets were found. Gene ontology enrichment analysis revealed that the adenylate cyclase-inhibiting G-protein coupled acetylcholine receptor signaling pathway was the key biological processes and were regulated simultaneously by the 17 direct targets. The KEGG pathway enrichment analysis found that three signaling pathways were closely related to AD prevention and treatment by QYG, including PI3K-Akt signaling pathway, regulation of actin cytoskeleton pathway and insulin resistance pathway.

Conclusion: This study demonstrated that QYG exerted the effect of preventing and treating $A D$ by regulating multi-targets with multi-components. Furthermore, the study demonstrated that a network pharmacology-based approach was useful for elucidation of the interrelationship between complex diseases and interventions of Chinese herbal medicines.

Keywords: Alzheimer disease, Gene regulatory networks, Genetic testing, Medicine, Chinese traditional, Molecular mechanisms of pharmacological action

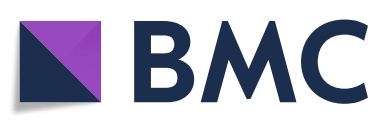

() The Author(s). 2020 Open Access This article is licensed under a Creative Commons Attribution 4.0 International License, which permits use, sharing, adaptation, distribution and reproduction in any medium or format, as long as you give appropriate credit to the original author(s) and the source, provide a link to the Creative Commons licence, and indicate if changes were made. The images or other third party material in this article are included in the article's Creative Commons licence, unless indicated otherwise in a credit line to the material. If material is not included in the article's Creative Commons licence and your intended use is not permitted by statutory regulation or exceeds the permitted use, you will need to obtain permission directly from the copyright holder. To view a copy of this licence, visit http://creativecommons.org/licenses/by/4.0/. The Creative Commons Public Domain Dedication waiver (http://creativecommons.org/publicdomain/zero/1.0/) applies to the data made available in this article, unless otherwise stated in a credit line to the data. 


\section{Introduction}

Alzheimer disease (AD), is a chronic neurodegenerative disease associated with the gradual degeneration of various cortical areas and it is also the cause of $60-70 \%$ of cases of dementia [1, 2]. In 2015, approximately 29.8 million people worldwide suffered from AD and dementia led to about 1.9 million deaths [1, 3]. The cause of Alzheimer's disease is complex and poorly understood, mainly including late age and the inheritance. Except that, a history of head injuries, depression and hypertension, smoking, obesity, diabetes and low level of education also increase the risks of AD. Although substantial progress has been made over the past few decades in exploring the pathophysiology and management of $\mathrm{AD}$, unfortunately, no recognized and feasible treatments have been found to prevent and cure AD. A few drugs for AD therapy affirmed by the US Food and Drug Administration (FDA), such as acetylcholinesterase (AChE)

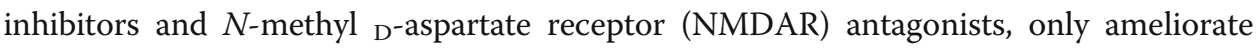
some symptoms of $\mathrm{AD}$, but they have no effect on the prevention and progression [4]. Therefore, it is urgent to systematically elucidate the mechanism of AD and search for safe and effective agents against AD.

Qiong Yu Gao (QYG), a classic anti-aging prescription, is consisted of Rehmanniae Radix (Sheng Dihuang), Poria Cocos (Fuling), Ginseng Radix et Rhizoma (Renshen) and MEL (Baimi). Rehmanniae Radix is the root tuber of Rehmannia glutinosa Libosch and is also one of the common and classic Chinese Medicine, which has been used in clinical practice for more than 3000 years in China. Traditionally, Rehmannia Radix is used for clearing heat and cooling blood, and nourishing Yin and promoting the production of body fluid. Modern researches further demonstrated the effects of Rehmannia Radix in treating fever, anemia, osteoporosis, hypertension, antioxidation and the disorder of autonomic nervous system [5-7]. Poria Cocos is the dried sclerotium of Poria Cocos (Schw.) Wolf and its traditional efficacy is eliminating dampness and diuresis, invigorating spleen and calming heart. Recent studies have shown that Poria Cocos exhibits anti-inflammatory, anti-cancer, antioxidant and antiviral activities [810]. Ginseng Radix et Rhizoma, the root and rhizome of Panax ginseng C. A. Mey, is very popular and well accepted all around the world. In china, Ginseng Radix et Rhizoma not only can be used alone for saving lives by reinforcing vital energy, but only plays an irreplaceable role in plenty of formulas such as Sijunzi decoction, Buzhongyiqi decoction, etc. [11] MEL is the honey of Apis cerana Fabricius and is commonly used for improving immune function, delaying aging and benefiting beauty [12, 13]. Actually, all of the four ingredients in QYG belong to medicinal and edible food/medicine. QYG has been clinically used for the treatment of cough with deficiency of yin and antiaging in China for over 700 years. Besides, according to the record in Compendium of Materia Medica, QYG could increase intelligence, make hair black and delay aging. And the same efficacy of QYG is also recorded in the ancient and classis book "Hong's Collection of Effective Recipes". Modern pharmacological study further demonstrated its anti-aging effect and complex mechanism, that QYG plays an important role in protecting the central nervous system of aging animals by effectively removing free radicals from the brain and activating mitochondrial autophagy in order to promote the repair of damaged neurons [14-16]. As aging is the leading risk factor for sporadic AD and the mechanism of anti-aging of QYG is closed related to pathophysiology of AD [17], therefore, both the ancient classical and modern evidences demonstrate that QYG is an 
effective agent for $\mathrm{AD}$ prevention and treatment, and a holistic understanding of its molecular mechanisms needs to be further explored.

Network pharmacology, as one part of Bioinformatics, was first proposed by Andrew L Hopkins [18]. Network pharmacology has been used in the modern research of Chinese Medicine in recent years, as it not only integrates biological information and systematic medicine, but also is in accordance with the connotation of holistic theory, multi-components and multi-targets of Chinese Medicine. Network pharmacology can potentially reveal mechanisms of complex herbal formulae by discovering bioactive ingredients and biomarkers.

In this study, network pharmacology was employed to establish the compoundtarget-disease network to explore the potential mechanism of QYG in AD prevention and treatment. The flowchart of the whole study design was illustrated in Fig. 1, and the following was a brief description: firstly, we collected the chemical ingredients in QYG and significant genes for AD; then target fishing was used to predict the potential targets and the compound-target-disease network was constructed; Finally, we conducted gene ontology enrichment analysis. This study may also contribute to the new function discovery and product development of QYG.

\section{Materials and methods}

\section{Collection of chemical ingredients in QYG}

All of the chemical ingredients of QYG were collected from Traditional Chinese Medicine Systems Pharmacology (TCMSP) Database [19] (http://lsp.nwu.edu.cn/tcmsp.php, designed by Center for Bioinformatics, Northwest University, Xian, Shaanxi, China), a Bioinformatics Analysis Tool for Molecular Mechanism of Traditional Chinese

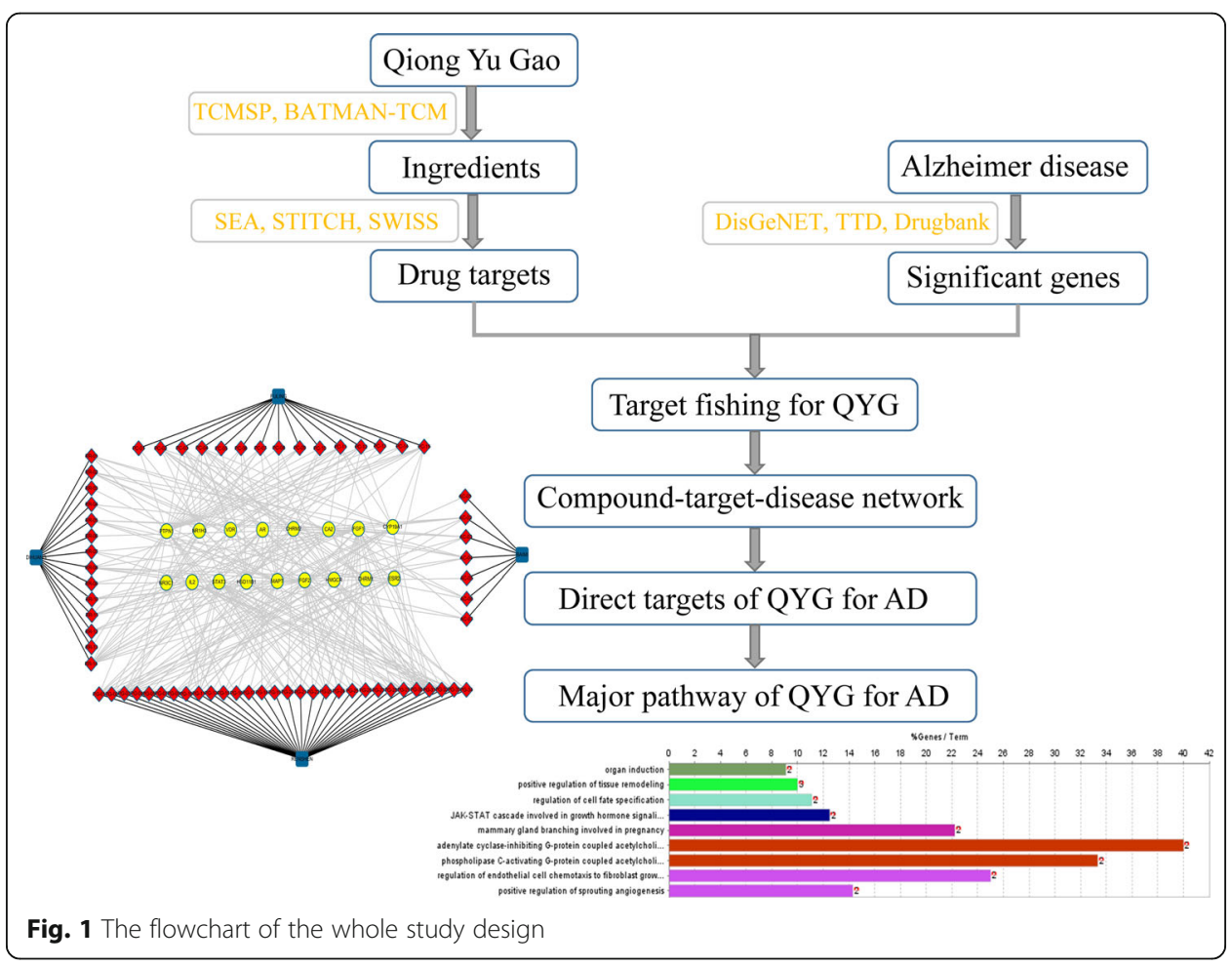


Medicine [20] (BATMAN-TCM, http://bionet.ncpsb.org/batman-tcm/, designed by State Key Laboratory of Proteomics, Beijing Proteome Research Center, Beijing Institute of Radiation Medicine, Beijing, China) and Traditional Chinese Medicine Integrated Database [21] (TCMID, http://www.megabionet.org/tcmid/, designed by Center for Bioinformatics and Computational Biology, The Institute of Biomedical Sciences, College of Life Science, East China Normal University, Shanghai, China). According to a suggested criterion in TCMSP database, the ingredients whose drug-likeness (DL) values $\geq 0.18$, oral bioavailability $(\mathrm{OB}) \geq 30 \%$ and blood-brain barrier $(\mathrm{BBB}) \geq-0.3$ were regarded as putative major ingredients and retained. In addition, those compounds with high contents and significant pharmacological activities but not meet the requirements were also retained.

\section{Significant genes collection for AD}

AD significant genes were retrieved from 3 databases, DisGeNET [22] Therapeutic Target Database (TTD, http://www.disgenet.org/home/, designed by Integrative Biomedical Informatics Group, Research Unit on Biomedical Informatics, Barcelona Biomedical Research Park, Barcelona, Spain) and Drugbank Database [23] (https://www.drugbank.ca/, created by the scientists at the University of Alberta, Edmonton, Canada). The repetitive genes collected from the two databases were removed.

\section{Target fishing for QYG}

The interaction between compounds and targets is the first principle for drug discovery. This target fishing was used to search for or predict the potential targets of small molecules. The targets of ingredients were predicted by Similarity Ensemble Approach [24] (SEA, http://sea.bkslab.org/, provided by the Shoichet Laboratory, Department of Pharmaceutical Chemistry, University of California, San Francisc, USA), STITCH [25] (http:// stitch.embl.de/, supported by European Molecular Biology Laboratory, Swiss Institute of Bioinformatics, NNF Center for Protein Research) and Swiss Target Prediction [26] (Swiss, http://www.swisstargetprediction.ch/, provided by Molecular Modelling Group, the University of Lausanne, Lausanne, Switzerland). Homo sapiens were the only species for the targets. The common targets between $\mathrm{AD}$ and small molecules were retained.

\section{Network construction and analysis}

The compound-target-disease network was constructed using Cytoscape software (Version 3.2.1, designed by Department of Bioengineering, University of California-San Diego, California, USA) [27]. The network was analyzed using Cytoscape plugin CentiScaPe to calculate topological parameters, mainly including the degree, betweenness centrality, closeness centrality and average shortest path length. The major ingredients and targets were represented by significant node, and the interactions were encoded by edges.

\section{Gene ontology enrichment analysis}

The KEGG pathway enrichment was carried out by the Database for Annotation, Visualization and Integrated Discovery (DAVID) [28]. The direct targets of QYG for AD treatment were input in the DAVID gene list panel, and the most significant 
pathways were obtained by analyzing the corresponding parameter value ( $p$ value). Besides, Cytoscape plugin ClueGO [29] was applied to analyze the gene ontology (GO) enrichment. The terms with Expression Analysis Systematic Explorer scores of $\leq 0.05$ were selected for functional annotation clustering and a $p$ value of $\leq 0.05$ for multiple hypothesis testing error was regarded as the significance cutoff [30]. Smaller $p$ values indicated greater enrichment.

\section{Results}

Candidate genes associated with AD

A total of 1932 significant genes were collected from the TTD and Drugbank databases after deleting repetitions (Supplementary file, Table S1).

\section{Putative major chemical ingredients in the 4 herbs of QYG}

Twenty-eight ingredients with $\mathrm{OB} \geq 30 \%, \mathrm{DL} \geq 0.18$ and $\mathrm{BBB} \geq-0.3$ from TCMSP, BATMAN-TCM and TCMID were selected. Besides, some compounds out of the condition but with pharmacological activities and high contents, including catalpol, rehmaglutin A, rehmaglutin B, rehmaglutin D, adenosine, acteoside, daucosterol, echinacoside, martynoside, rehmaionoside $B$ and rehmaionoside $C$ from Rehmanniae Radix (RR), polyporenic acid C, pachymic acid, poricoic acid A, poricoic acid B, poricoic acid C, poricoic acid D, poricoic acid DM, tumulosic acid and ergosterol from Poria Cocos (PC), ginsenoside Rh2, panaxynol, 20(S)-protopanaxadiol, ginsenoside La, ginsenoside Ro, ginsenoside Rc, ginsenoside Re, ginsenoside rf, gypenoside LXIX, sanchinoside C1, Panaxytriol, 20(R)-ginsenoside Rg2, 20-(S)-Ginsenoside Rg3, ginsenoside $\mathrm{Rb} 1$, and ginsenoside Rg1 from Ginseng Radix et Rhizoma of Panax ginseng C. A. Mey (PG), and fructose, glucose, maltose, pyridoxine, acetylcholine and sucrose from MEL of Apis cerana (AC), were also regarded as putative active ingredients. The final candidate compounds were shown in Supplementary file, Table S2.

\section{Network construction of the anti-AD targets of QYG}

According to pharmacophore matching, some statistical factors and similarity measures, 1270 targets of QYG for Homo sapiens were obtained. In which, RR contained 333, PC contained 184, PG contained 594, and AC contained 159 (Supplementary file, Table S3). And compound-target-disease network for the 4 herbs were constructed, respectively (RR-Targets, Fig. 2a; PC-Targets, Fig. 2b; PG-Targets, Fig. 2c and ACTargets, Fig. 2d). The results revealed that 14 major ingredients in RR, 15 in PC, 34 in PG and 7 in AC may play important roles in anti-AD.

As shown in Fig. 2a, 123 AD-related targets were associated with 14 ingredients in $R R$ and the analysis of network revealed that $\beta$-sitosterol (41 targets) was predicted as the major ingredient in RR. FGF1 and FGF2 were forecasted as the major targets of RR for the treatment of AD. As shown in Fig. 2b, the PC network included 93 targets. Among the ingredients of PC, polyporenic acid C (48 targets) was regarded as a major ingredient. PTPN1, HSD11B1, NR3C1, POLB and NR1H3 were predicted as major targets of PC for the treatment of AD. The PG network was shown in Fig. 2c, containing 240 targets. Gomisin A (61 targets) was predicted as the major ingredient of PG for the treatment of AD, and FGF1, FGF2, VEGFA and STAT3 were predicted as the major 


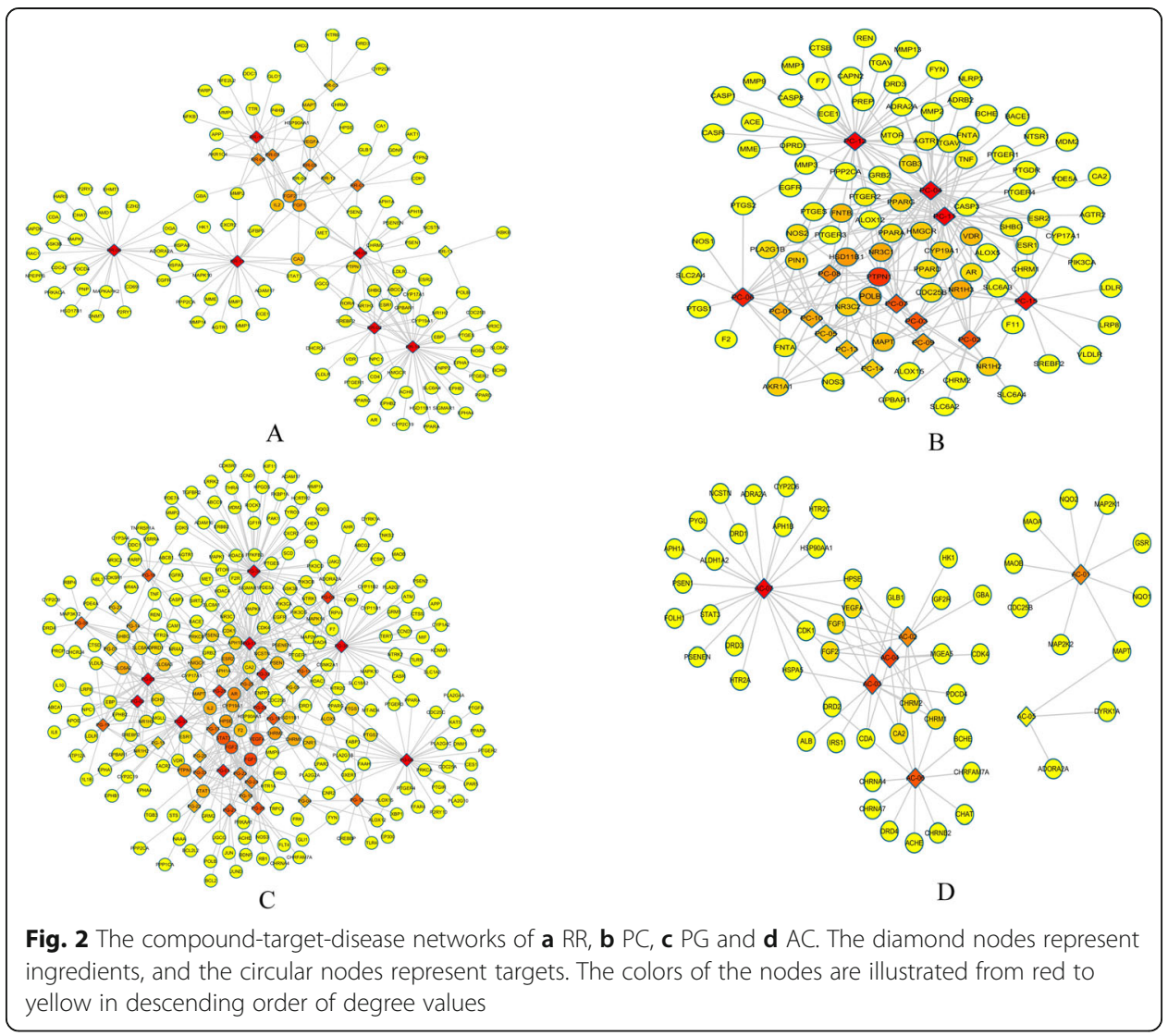

targets. For AC network, 7 bioactive ingredients in $\mathrm{AC}$ were validated to bind with 55 AD-related targets, in which sucrose (23 targets) was forecasted as the major ingredient and CA2, FGF1, CHRM2, FGF2, CHRM1 and VEGFA were the major target (Fig. 2d).

As shown in Fig. 3, the compound-target-disease interaction network was constructed. The ingredients of QYG were described by diamond nodes, and the direct targets of QYG for AD treatment were encoded by circular nodes. Only the targets with higher values of "degree" (above two-fold of the median value) "betweenness centrality" and "closeness centrality" (above the median value), and "average shortest path length" (below the median value) were identified as the candidate targets of QYG for AD. Ultimately, 17 direct targets were screened (Table 1).

\section{The characteristics of QYG in the treatment of $A D$}

As shown in Fig. 4, the ingredients directly to the targets were obtained via the compound-target-disease interaction network. Multi-ingredients from RR, PC, PG and $\mathrm{AC}$ regulated almost all direct targets. The results indicated that the anti-AD effect of QYG was played through regulating multi-targets by multi-ingredients.

\section{GO enrichment analysis for targets}

To investigate the biological functions of the direct targets of QYG for AD, the gene GO biological process (BP) was performed by the Cytoscape plugin ClueGO. As shown in Fig. 5, 9 most significantly enriched GOBP terms $(p \leq 0.05)$ were listed. Among the 9 


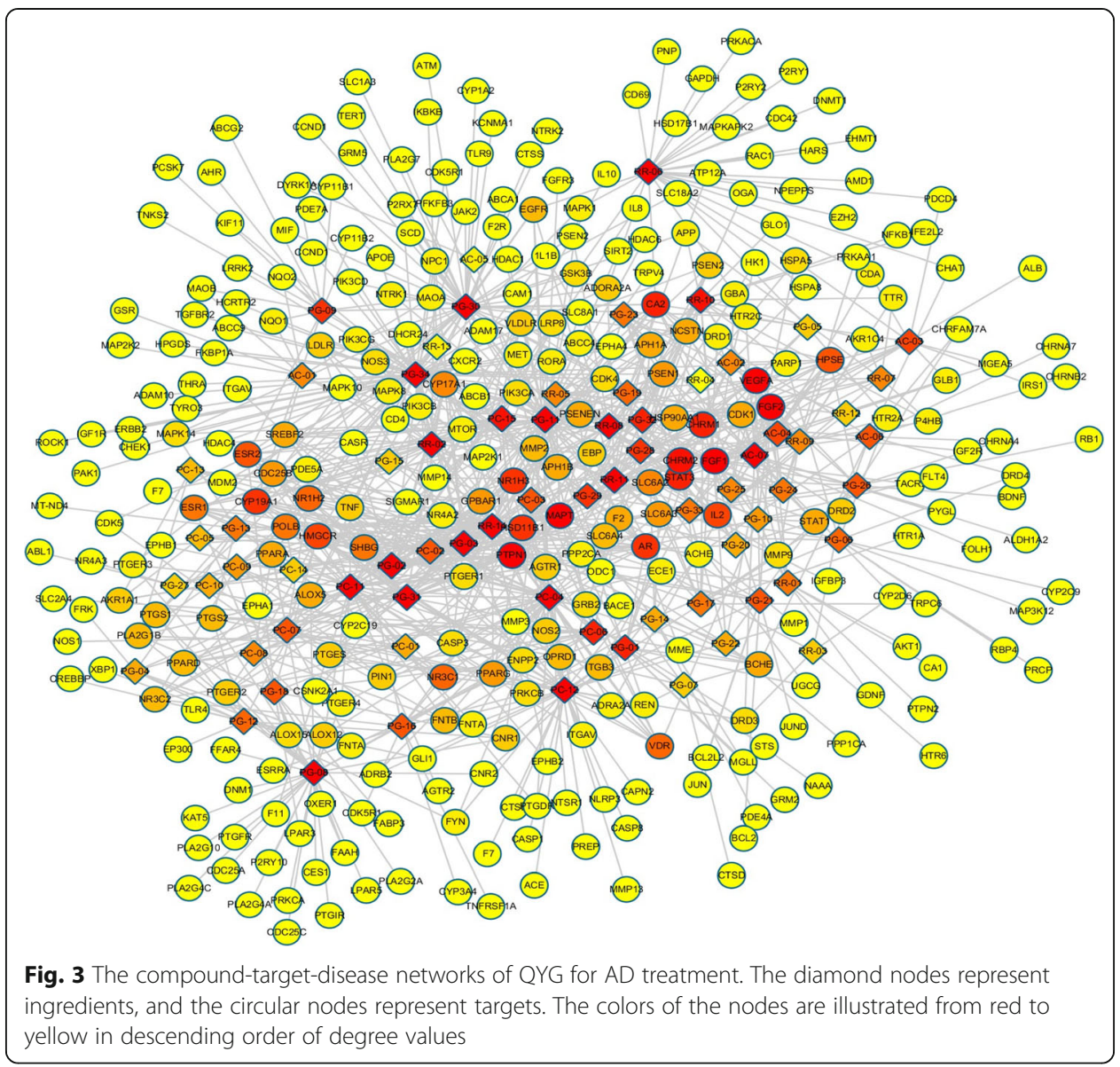

GOBP terms, adenylate cyclase-inhibiting G-protein coupled acetylcholine receptor signaling pathway was the most prominent.

\section{Target-pathway network and analysis}

The functional annotation tool of DAVID was used for KEGG pathway enrichment analysis. Ultimately, three signaling pathways were obtained, including PI3K-Akt signaling pathway, regulation of actin cytoskeleton pathway and insulin resistance pathway (Table 2).

\section{Discussion}

The etiology and pathogenesis of $\mathrm{AD}$ is complex. Chinese medicine, focus on systemic functional adjustments, is more suitable for the disease with complex pathogenesis. QYG, as a TCM formula, has the characteristics of multi-components and multitargets therapy for AD. However, it is difficult to reveal its possible active ingredients and pharmacological mechanism. Network pharmacology may be an excellent method, which contributes to collecting active ingredients and pharmacological actions of herbs or TCM formula. In this study, the network pharmacology approach was used to identify the putative bioactive compounds in QYG and potential targets, and to explore the potential mechanisms of QYG for AD prevention and treatment. The results showed 
Table 1 The major targets of QYG for AD treatment and its relevant topological parameters

\begin{tabular}{|c|c|c|c|c|c|c|}
\hline $\begin{array}{l}\text { Uniprot } \\
\text { ID }\end{array}$ & Protein name & $\begin{array}{l}\text { Gene } \\
\text { name }\end{array}$ & Degree & $\begin{array}{l}\text { Betweenness } \\
\text { centrality }\end{array}$ & $\begin{array}{l}\text { Closeness } \\
\text { centrality }\end{array}$ & $\begin{array}{l}\text { Average shortest } \\
\text { path length }\end{array}$ \\
\hline P18031 & $\begin{array}{l}\text { Tyrosine-protein phosphatase } \\
\text { non-receptor type } 1\end{array}$ & PTPN1 & 26 & 0.066106 & 0.337931 & 2.959184 \\
\hline P05230 & Fibroblast growth factor 1 & FGF1 & 25 & 0.041648 & 0.342657 & 2.918367 \\
\hline P09038 & Fibroblast growth factor 2 & FGF2 & 24 & 0.033146 & 0.334471 & 2.989796 \\
\hline P10636 & $\begin{array}{l}\text { Microtubule-associated protein } \\
\text { tau }\end{array}$ & MAPT & 18 & 0.038501 & 0.309637 & 3.229592 \\
\hline P08172 & $\begin{array}{l}\text { Muscarinic acetylcholine } \\
\text { receptor M2 }\end{array}$ & CHRM2 & 16 & 0.02588 & 0.319739 & 3.127551 \\
\hline P00918 & Carbonic anhydrase 2 & CA2 & 15 & 0.069146 & 0.345679 & 2.892857 \\
\hline P11229 & $\begin{array}{l}\text { Muscarinic acetylcholine } \\
\text { receptor M1 }\end{array}$ & CHRM1 & 15 & 0.026518 & 0.324503 & 3.081633 \\
\hline P40763 & $\begin{array}{l}\text { Signal transducer and activator } \\
\text { of transcription } 3\end{array}$ & STAT3 & 15 & 0.011336 & 0.315113 & 3.173469 \\
\hline P10275 & Androgen receptor & $A R$ & 14 & 0.037305 & 0.360958 & 2.770408 \\
\hline P11511 & Cytochrome P450 19A1 & CYP19A1 & 14 & 0.024461 & 0.332767 & 3.005102 \\
\hline P28845 & $\begin{array}{l}\text { Corticosteroid 11-beta- } \\
\text { dehydrogenase isozyme } 1\end{array}$ & HSD11B1 & 14 & 0.029686 & 0.345679 & 2.892857 \\
\hline Q13133 & Oxysterols receptor LXR-alpha & $\mathrm{NR} 1 \mathrm{H} 3$ & 14 & 0.008421 & 0.311111 & 3.214286 \\
\hline P04035 & $\begin{array}{l}\text { 3-hydroxy-3-methylglutaryl- } \\
\text { coenzyme A reductase }\end{array}$ & HMGCR & 13 & 0.030442 & 0.34087 & 2.933673 \\
\hline P60568 & Interleukin-2 & IL2 & 13 & 0.011053 & 0.316129 & 3.163265 \\
\hline Q92731 & Estrogen receptor beta & ESR2 & 12 & 0.016322 & 0.329412 & 3.035714 \\
\hline P04150 & Glucocorticoid receptor & NR3C1 & 11 & 0.019069 & 0.331641 & 3.015306 \\
\hline P11473 & Vitamin D receptor & VDR & 11 & 0.007378 & 0.311111 & 3.214286 \\
\hline
\end{tabular}

that QYG exerted the effect of treating AD by regulating multi-targets with multicomponents.

Specifically, 70 ingredients of these 4 herbs were screened out. In which, several ingredients have been reported to improve the cognitive impairment and neurodegenerative disorders. For example, catalpol in RR is used for improving learning ability and memory for AD patients [31]. Trametenolic acid could significantly improve learning and memory ability of the cerebral ischemia injury rats [32]. Wu et al. found that Hederagenin could be used as a novel autophagic enhancer to play a role in neuroprotection by improving motor deficits in Parkinson's disease mice model [33]. Stigmasterol could ameliorate memory impairment and promotes neuritogenesis and synaptogenesis [34]. Ginsenosides from ginseng showed the great therapeutic potential in $\mathrm{AD}$ [35]. The above evidence suggests the activities of these ingredients in QYG for the treatment of $\mathrm{AD}$, but further experimental verification is still needed.

By target fishing for QYG and AD, the result showed that ingredients affected 123 AD-related targets in RR, 93 in PC, 240 in PG and 55 in AC. Furthermore, 17 representative targets including PTPN1, FGF1, FGF2, MAPT, CHRM2, CA2, CHRM1, STAT3, AR, CYP19A1, HSD11B1, NR1H3, HMGCR, IL2, ESR2, NR3C1 and VDR were regulated QYG. In which MAPT, CHRM1, CHRM2 and CA were the overlapped targets of the four herbs in QYG, reflecting that some active ingredients may interact with each other to play a synergistic effect. The relevant literatures also revealed that FGF1, FGF2, MAPT, CHRM2, CA2, CHRM1, STAT3, AR, CYP19A1, HSD11B1, NR1H3, 


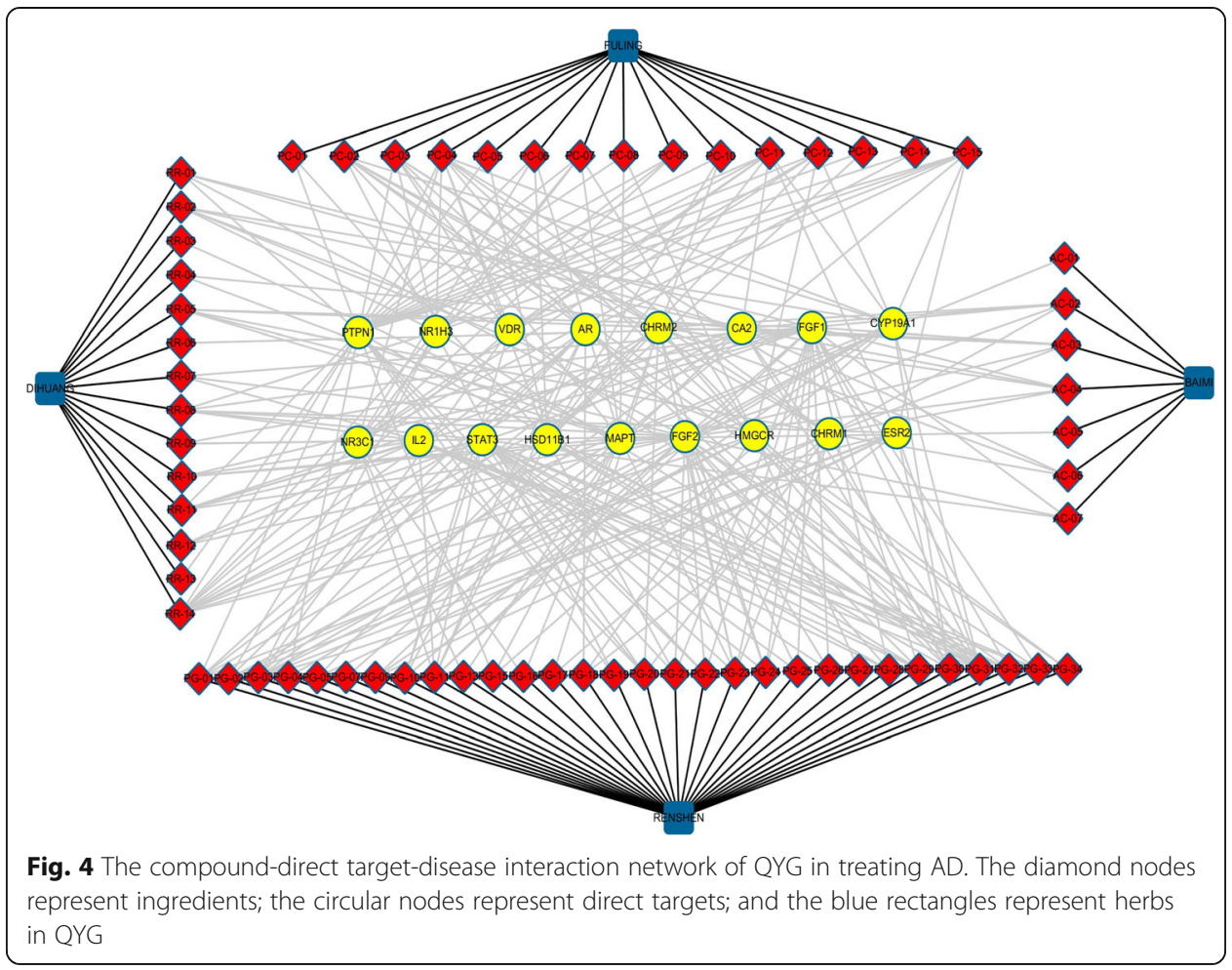

HMGCR, ESR2, ESR1 and VDR may be involved in the pathogenesis of AD. The results of GO enrichment analysis revealed that the direct targets of QYG for AD were closely related to the adenylate cyclase-inhibiting G-protein coupled acetylcholine receptor signaling pathway.

Putative bioactive compounds in QYG associated with direct AD genes were placed in the DAVID for KEGG pathway analysis. According to the corresponding parameter ( $p$ value), some important pathways that may be regulated by QYG in the process of treating diseases were screened out, including PI3K-Akt signaling pathway, regulation of actin cytoskeleton pathway and insulin resistance pathway. The actin cytoskeleton is a dynamic network made up of actin polymers and associated actin binding proteins. Recent studies found that interference with the expression or function of upstream regulators of the actin cytoskeleton, including Rac-GEFs, Rac, and Rac targets, PAK could cause spine and synapse loss [36-38]. Interestingly, synapse degeneration is also

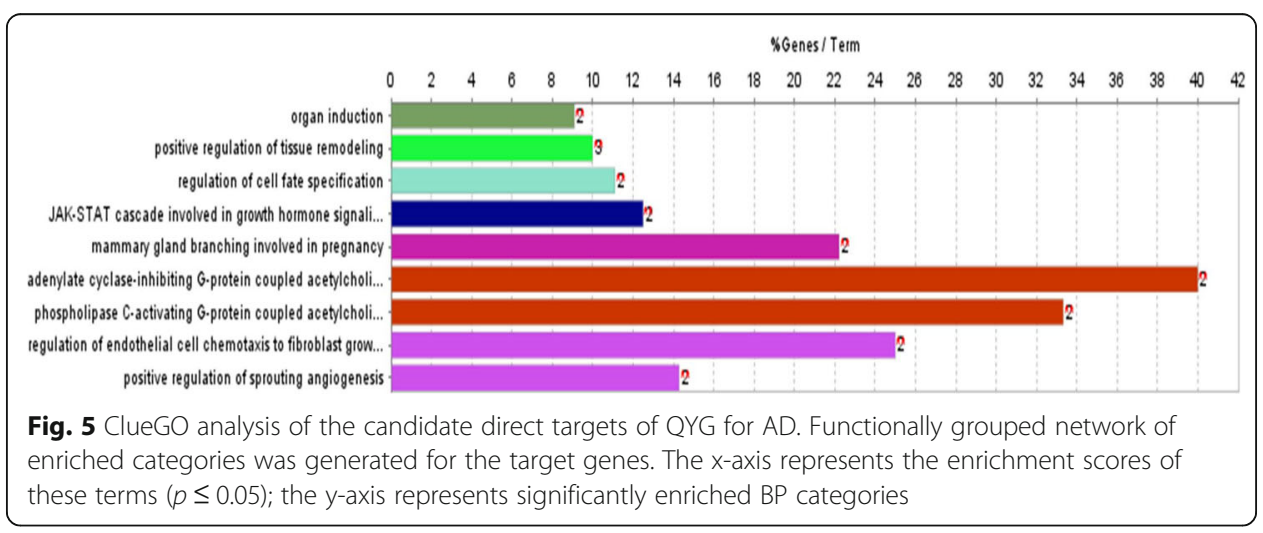


Table 2 The KEGG pathway annotation of the direct targets of QYG for AD treatment

\begin{tabular}{llll}
\hline Signaling pathway & Count & $\boldsymbol{p}$ value & Gene \\
\hline PI3K-Akt signaling pathway & 5 & 0.0070 & FGF2, IL2, CHRM2, CHRM1, FGF1 \\
Regulation of actin cytoskeleton pathway & 4 & 0.0117 & FGF2, CHRM2, CHRM1, FGF1 \\
Insulin resistance pathway & 3 & 0.0254 & NR1H3, PTPN1, STAT3 \\
\hline
\end{tabular}

demonstrated as a major component of AD pathology [39]. PI3K/Akt pathway is a signalling cascade centering on serine/threonine kinase Akt. Misregulation of the PI3K/ AKT signaling pathway has been found to be closely associated with AD. The PI3KAkt signaling pathway in $\mathrm{AD}$ is involved in regulating many basic cellular functions, such as cell cycle growth, cell migration, and apoptosis [40]. The insulin signal pathway mediating normal biological effects mainly include insulin receptor, insulin receptor substrates, phosphatidylinositol-3 kinase, phosphorylated PI3K, serine-threonine kinase, and phosphorylated AKT [41]. And emerging data have demonstrated that ADassociated abnormalities in energy metabolism is caused by brain insulin resistance and insulin deficiency. In turn, AD can further result in brain insulin signaling pathway injuries. In these pathways, genes CHRM2, CHRM1 are involved in three pathways. CHRM1 belongs to excitatory M1 class of muscarinic acetylcholine receptor and CHRM2 belongs to inhibitory M2 class. Increasing evidence suggest that CHRM1 may be a candidate gene for the pathogenesis of AD, with decreased CHRM1 or CHRM1 mRNA level, or unchanged central CHRM1 levels, but with the reduced radioligandbinding affinity $[42,43]$. CHRM2 gene is demonstrated to be involved in synaptic plasticity, neuronal excitability, release of acetylcholine and cognitive function [44, 45]. And studies found that neuritic plaques, neurofibrillary tangles, soluble amyloid, neuronal death, loss of neurotransmitters, synapse loss, inflammation, and oxidative stress may be the targets for primary prevention in AD [46].

This study systematically explored the putative bioactive compounds in QYG and pharmacological mechanisms of QYG for AD prevention and treatment through network pharmacology. QYG was selected based on our previous study, which was much more reliable than those based on literature retrieval. All the ingredients of QYG belongs to medicinal and edible plant or diet, and they have a history of safe use. The potential advantage of "network targets, multi-component" strategy of network pharmacology is obvious, but it is generally as a guided theoretical science, which is also the limitation of this study. Therefore, the demonstration in the gene and protein levels of QYG treated Alzheimer disease will be provided in the future.

\section{Conclusion}

This study demonstrated that the network pharmacological analysis was useful for elucidation of the interrelationship between complex diseases such as AD and interventions of Chinese medicines. Based on the study, we analyzed the active materials and the possible molecular mechanism of QYG on AD, which will also provide a theoretical basis for the future in-depth study of QYG for AD treatment. Besides, as QYG belongs to medicinal and edible formula, it will have a broader clinical practice and market. 


\section{Supplementary information}

Supplementary information accompanies this paper at https://doi.org/10.1186/s13040-020-00212-z.

Additional file 1 : Table S1. The 1981 significant genes associated with AD. Table S2. The putative major chemical ingredients and ADME parameters in QYG. Table S3. The detailed target information of the ingredients of herbs

\section{Abbreviations}

AD: Alzheimer disease; QYG: Qiong Yu Gaö FDA: US Food and Drug Administration; AChE: Acetylcholinesterase; NMDAR: N-methyl D-aspartate receptor; TCMSP: Traditional Chinese Medicine Systems Pharmacology; BATMANTCM: Bioinformatics Analysis Tool for Molecular mechanism of Traditional Chinese Medicine; DL: Drug-likeness; OB: Oral bioavailability; DAVID: Annotation, Visualization and Integrated Discovery; GO: Gene ontology; BP: Biological process; RR: Radix Rehmanniae; PC: Poria Cocos (Schw.) Wolf; PG: Panax ginseng C. A. Mey; AC: Apis cerana

\section{Acknowledgements}

The study was supported by China Postdoctoral Science Foundation (2018 M633037).

\section{Authors' contributions}

YJS and CW: study concept and design; acquisition of data; analysis and interpretation of data; drafting of the manuscript; critical revision of the manuscript for important intellectual content; statistical analysis. WXL and HLJ: acquisition of data; analysis and interpretation of data; added experiment results. LCY, LRK, GF and ZMY: material support; analysis and interpretation of data; critical revision of the manuscript. LHL and QWL: revision of the manuscript and study supervision. The author(s) read and approved the final manuscript.

\section{Availability of data and materials}

All data are available in the manuscript and they are showed in figures, tables and supplement file.

\section{Competing interests}

The authors declare that they have no conflict of interest.

\section{Author details}

${ }^{1}$ School of Basic Medical Sciences, Guangzhou University of Chinese Medicine, Guangzhou, Guangdong Province, China. ${ }^{2}$ The First Affiliated Hospital of Guangzhou University of Chinese Medicine, Guangzhou, Guangdong Province, China. ${ }^{3}$ Department of Pharmacology and Pharmacy, The University of Hong Kong, Pok Fu Lam, Hong Kong. ${ }^{4}$ College of Pharmacy, Chengdu University of Traditional Chinese Medicine, Chengdu, Sichuan Province, China. ${ }^{5}$ Division of Hematology, Renji Hospital, School of Medicine, Shanghai Jiaotong University, Shanghai, China.

Received: 11 February 2020 Accepted: 15 April 2020

Published online: 25 April 2020

\section{References}

1. World Health Organization endorses global action plan on rising incidence of dementia. Nurs Older People. 2017;29:7

2. Guarino A, Favieri F, Boncompagni I, Agostini F, Cantone M, Casagrande M. Executive functions in Alzheimer disease: a systematic review. Front Aging Neurosci. 2018;10:437.

3. Mortality GBD, Causes of Death C. Global, regional, and national life expectancy, all-cause mortality, and cause-specific mortality for 249 causes of death, 1980-2015: a systematic analysis for the global burden of disease study 2015. Lancet. 2016:388:1459-544.

4. Masoumi J, Abbasloui M, Parvan R, et al. Apelin, a promising target for Alzheimer disease prevention and treatment. Neuropeptides. 2018;70:76-86.

5. Bao BH, Qian Y, Cheng FF, et al. A novel integrative processing Technology for the Preparation of Rehmanniae Radix slices. Evid Based Complement Alternat Med. 2018;2018:4524797.

6. Chao CH, Hsu JL, Chen MF, et al. Anti-hypertensive effects of Radix Rehmanniae and its active ingredients. Nat Prod Res. 2018:12:1-6.

7. Xue S, Wang L, Chen S, Cheng Y. Simultaneous analysis of saccharides between fresh and processed Radix Rehmanniae by HPLC and UHPLCLTQ-Orbitrap-MS with multivariate statistical analysis. Molecules. 2018;23:541.

8. Lee $\mathrm{S}$, Choi E, Yang SM, et al. Bioactive compounds from sclerotia extract of Poria cocos that control adipocyte and osteoblast differentiation. Bioorg Chem. 2018;81:27-34.

9. Wang N, Zhang Y, Wang X, et al. Antioxidant property of water-soluble polysaccharides from Poria cocos wolf using different extraction methods. Int J Biol Macromol. 2016;83:103-10.

10. Sun Y. Biological activities and potential health benefits of polysaccharides from Poria cocos and their derivatives. Int J Biol Macromol. 2014;68:131-4.

11. Zhang L, Liu XY, Xu W, Yang XW. Pharmacokinetics comparison of 15 ginsenosides and 3 aglycones in ginseng Radix et Rhizoma and Baoyuan decoction using ultra-fast liquid chromatography coupled with triple quadrupole tandem mass spectrometry. Phytomedicine. 2019;59:152775.

12. Behrends A, Scheiner R, Baker N, Amdam GV. Cognitive aging is linked to social role in honey bees (Apis mellifera). Exp Gerontol. 2007:42:1146-53.

13. Margotta JW, Mancinelli GE, Benito AA, Ammons A, Roberts SP, Elekonich MM. Effects of flight on gene expression and aging in the honey bee brain and flight muscle. Insects. 2012;4:9-30.

14. Qu WL, Cao Y, Liu HL, Tong L, Fan R, Liu RF. Target protein candidates of hypothalamus in aging rats with intervention by Qiongyugao. Zhongguo Zhong Yao Za Zhi. 2016;41:1308-12. 
15. Xue YFLZ. Effects of Qiong-yu-gao on the nervous system in a mouse model of aging. Tradit Chin Drug Res \& Clin Pharmacolo. 1999;10:159-61..

16. Zhang Q, Xie Q, Chen X, Sun L, Ye K, Tang C. Effect of qiongyugao on the action of DDP in inhibiting the division of GLC-82 cell strain in vitro. Zhong Yao Cai. 2000;23:694-6.

17. YU YT LH, Li QX, Liu RF. Qiongyu ointment for the prevention and treatment of Alzheimer disease. Jilin J Tradit Chin Med. 2014;34:931-4.

18. Hopkins AL. Network pharmacology: the next paradigm in drug discovery. Nat Chem Biol. 2008;4:682-90.

19. Ru J, Li P, Wang J, et al. TCMSP: a database of systems pharmacology for drug discovery from herbal medicines. J Cheminform. 2014;6:13.

20. Liu Z, Guo F, Wang Y, et al. BATMAN-TCM: a bioinformatics analysis tool for molecular mechANism of traditional Chinese medicine. Sci Rep. 2016;6:21146.

21. Xue R, Fang Z, Zhang M, Yi Z, Wen C, Shi T. TCMID: traditional Chinese medicine integrative database for herb molecular mechanism analysis. Nucleic Acids Res. 2013;41:D1089-95.

22. Pinero J, Bravo A, Queralt-Rosinach N, et al. DisGeNET: a comprehensive platform integrating information on human disease-associated genes and variants. Nucleic Acids Res. 2017:45:D833-D9.

23. Wishart DS, Feunang YD, Guo AC, et al. DrugBank 5.0: a major update to the DrugBank database for 2018. Nucleic Acids Res. 2018:46:D1074-D82.

24. Keiser MJ, Roth BL, Armbruster BN, Ernsberger P, Irwin JJ, Shoichet BK. Relating protein pharmacology by ligand chemistry. Nat Biotechnol. 2007;25:197-206.

25. Szklarczyk D, Santos A, von Mering C, Jensen LJ, Bork P, Kuhn M. STITCH 5: augmenting protein-chemical interaction networks with tissue and affinity data. Nucleic Acids Res. 2016;44:D380-4.

26. Gfeller D, Grosdidier A, Wirth M, Daina A, Michielin O, Zoete V. SwissTargetPrediction: a web server for target prediction of bioactive small molecules. Nucleic Acids Res. 2014;42:W32-8.

27. Smoot ME, Ono K, Ruscheinski J, Wang PL, Ideker T. Cytoscape 2.8: new features for data integration and network visualization. Bioinformatics. 2011;27:431-2.

28. da Huang W, Sherman BT, Lempicki RA. Systematic and integrative analysis of large gene lists using DAVID bioinformatics resources. Nat Protoc. 2009:4:44-57.

29. Bindea G, Mlecnik B, Hackl H, et al. ClueGO: a Cytoscape plug-in to decipher functionally grouped gene ontology and pathway annotation networks. Bioinformatics. 2009;25:1091-3.

30. Huang J, Tang H, Cao S, et al. Molecular targets and associated potential pathways of Danlu capsules in hyperplasia of mammary glands based on systems pharmacology. Evid Based Complement Alternat Med. 2017;2017:1930598.

31. Cui Y, Yan Z, Hou S, Chang Z. Effect of radix Rehmanniae preparata on the expression of c-fos and NGF in hippocampi and learning and memory in rats with damaged thalamic arcuate nucleus. Zhong Yao Cai. 2004;27:589-92.

32. Wang J, Wang A, He H, et al. Trametenolic acid B protects against cerebral ischemia and reperfusion injury through modulation of microRNA-10a and PI3K/Akt/mTOR signaling pathways. Biomed Pharmacother. 2019;112:108692.

33. Wu AG, Zeng W, Wong VK, et al. Hederagenin and alpha-hederin promote degradation of proteins in neurodegenerative diseases and improve motor deficits in MPTP-mice. Pharmacol Res. 2017:115:25-44.

34. Haque MN, Moon IS. Stigmasterol upregulates immediate early genes and promotes neuronal cytoarchitecture in primary hippocampal neurons as revealed by transcriptome analysis. Phytomedicine. 2018;46:164-75.

35. Kim MS, Yu JM, Kim HJ, et al. Ginsenoside re and Rd enhance the expression of cholinergic markers and neuronal differentiation in Neuro-2a cells. Biol Pharm Bull. 2014;37:826-33.

36. Tashiro A, Minden A, Yuste R. Regulation of dendritic spine morphology by the rho family of small GTPases: antagonistic roles of Rac and rho. Cereb Cortex. 2000;10:927-38.

37. Cahill ME, Xie Z, Day M, et al. Kalirin regulates cortical spine morphogenesis and disease-related behavioral phenotypes. Proc Natl Acad Sci U S A. 2009;106:13058-63.

38. Penzes $P$, Beeser A, Chernoff J, et al. Rapid induction of dendritic spine morphogenesis by trans-synaptic ephrinB-EphB receptor activation of the rho-GEF kalirin. Neuron. 2003;37:263-74.

39. Selkoe DJ. Alzheimer's disease is a synaptic failure. Science. 2002;298:789-91.

40. Kitagishi Y, Matsuda S. Diets involved in PPAR and PI3KNAKT/PTEN pathway may contribute to neuroprotection in a traumatic brain injury. Alzheimers Res Ther. 2013;5:42.

41. Feng HL, Dang HZ, Fan $H$, et al. Curcumin ameliorates insulin signalling pathway in brain of Alzheimer's disease transgenic mice. Int J Immunopathol Pharmacol. 2016;29:734-41.

42. Rodriguez-Puertas R, Pascual J, Vilaro T, Pazos A. Autoradiographic distribution of M1, M2, M3, and M4 muscarinic receptor subtypes in Alzheimer's disease. Synapse. 1997;26:341-50.

43. Ladner CJ, Lee JM. Reduced high-affinity agonist binding at the M(1) muscarinic receptor in Alzheimer's disease brain: differential sensitivity to agonists and divalent cations. Exp Neurol. 1999;158:451-8.

44. Gosso FM, de Geus EJ, Polderman TJ, Boomsma DI, Posthuma D, Heutink P. Exploring the functional role of the CHRM2 gene in human cognition: results from a dense genotyping and brain expression study. BMC Med Genet. 2007;8:66.

45. Volpicelli LA, Levey Al. Muscarinic acetylcholine receptor subtypes in cerebral cortex and hippocampus. Prog Brain Res. 2004;145:59-66.

46. Thal LJ. Prevention of Alzheimer disease. Alzheimer Dis Assoc Disord. 2006;20:597-9.

\section{Publisher's Note}

Springer Nature remains neutral with regard to jurisdictional claims in published maps and institutional affiliations. 